\title{
New and old biomarkers of hepatocellular carcinoma
}

\author{
Georgios Zacharakis ${ }^{1,2}$, Ahmad Aleid ${ }^{3}$, Khaled K Aldossari $^{4}$ \\ 'Endoscopy Unit, Limassol General Hospital, St George's Medical School, University of Nicosia, Nicosia 1700, Republic of Cyprus. \\ 2Endoscopy Unit, Department of Internal Medicine, College of Medicine, Prince Sattam bin Abdulaziz University, University Hospital, \\ Al Kharj 16277, Saudi Arabia. \\ ${ }^{3}$ Department of Gastroenterology and Hepatology, King Fahad Medical City, Riyadh 11525, Saudi Arabia. \\ ${ }^{4}$ Department of Family Medicine, College of Medicine, Prince Sattam bin Abdulaziz University, University Hospital, Al Kharj 16277, \\ Saudi Arabia.
}

Correspondence to: Dr. Georgios Zacharakis, Endoscopy Unit, Department of Internal Medicine, Prince Sattam bin Abdulaziz University Hospital, Al Kharj 16277, Saudi Arabia. E-mail: GZacharakis@yahoo.gr

How to cite this article: Zacharakis G, Aleid A, Aldossari KK. New and old biomarkers of hepatocellular carcinoma. Hepatoma Res 2018;4:65. http://dx.doi.org/10.20517/2394-5079.2018.76

Received: 11 Jun 2018 First Decision: 24 Jul 2018 Revised: 12 Sep 2018 Accepted: 13 Sep 2018 Published: 17 Oct 2018

Science Editor: Guang-Wen Cao Copy Editor: Cui Yu Production Editor: Zhong-Yu Guo

\begin{abstract}
Hepatocellular carcinoma (HCC) is a significant cause of mortality in patients with chronic liver disease around the world. Development of biomarkers for early HCC detection is a primary public health goal to decrease mortality. The ideal biomarkers should be highly sensitive and specific for surveillance of high-risk populations and early detection of HCC and also be able to predict therapeutic outcome and provide a prognosis on survival. Currently, the new biomarkers do not perform better than the conventional ones such as alpha-fetoprotein in such a way that they could be widely adopted in clinical practice. Another problem is the low sensitivity of these biomarkers in the detection of HCC. Further work on the development of novel biomarkers and on a combination of them is necessary. Advances in identifying unique molecular signatures including genomic, proteomic, metabolomic, and glycomic profiles have improved our understanding of many biological processes involved in HCC. This review focuses on the role of old and new biomarkers in surveillance, diagnosis, prognosis, and prediction of response to therapeutic targets for HCC and provides up-to-date data to health-care providers which would be applied in clinical practice.
\end{abstract}

Keywords: Hepatocellular carcinoma, biomarkers, diagnosis, surveillance, prognosis, treatment response

\section{INTRODUCTION}

Hepatocellular carcinoma (HCC) is the second leading cause of cancer-related mortality and morbidity. HCC accounts for about $6 \%$ of all newly diagnosed cancer cases worldwide ${ }^{[1,2]}$. Risk factors include chronic hepatitis $\mathrm{B}$ virus (HBV) and hepatitis C virus (HCV) infection which contributes up to $85 \%$ of $\mathrm{HCC}$ cases worldwide ${ }^{[3]}$.

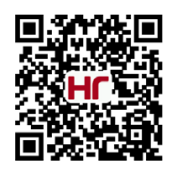


Other risk factors include metabolic disorders such as nonalcoholic fatty liver disease (NAFLD) and chronic alcohol consumption ${ }^{[4]}$. The frequency of cirrhosis among patients with HCC has been shown to be $85 \%$ $95 \%{ }^{[5,6]}$. The HCC incidence rate among cirrhotic patients has been estimated to be $2 \%-4 \%$ per year ${ }^{[7]}$. Cirrhotic patients represent a high-risk group for HCC development and should undergo surveillance for HCC on a regular basis.

Early detection of HCC through surveillance methods have increased patient survival by providing effective initial treatments such as primary curative hepatectomy and locoregional ablative therapy ${ }^{[8,9]}$. Surveillance and diagnostic methods for HCC depend on several biomarkers, defined as molecules that can be objectively measured in body fluids.

Although many studies have investigated several biomarkers for the prognosis and the evaluation of HCC, no biomarkers can predict and/or confirm the presence of HCC. There are no validated predictive biomarkers to evaluate the therapeutic response to HCC treatment except for the alpha-fetoprotein (AFP) for the evaluation of ramucirumab treatment efficacy as recently presented by Zhu et al. ${ }^{[10]}$.

The most well-studied HCC biomarkers are (1) the AFP, its isoform lens culinaris agglutinin-reactive fraction of alpha-fetoprotein (AFP-L3); and (2) des- $\gamma$-carboxy prothrombin (DCP). However, there are many other molecules that might be taken into account for future studies, including glypican 3 (GPC3), glutamine synthase (GS), heat shock protein 70 (HSP70), cytokeratin 19 (CK19), Golgi protein 73 (GP73), midkine, osteopontin (OPN), squamous cell carcinoma antigen (SCCA), Annexin A2, fibroblast growth factor 3/4 (FGF3/4), microRNAs (miRNAs), Long non-coding RNAs (lncRNAs), circulating tumor cells (CTCs), cell-free DNA (cfDNA), and other biomarkers based on proteomic analyses. In addition, genetic signatures might play a role in the prognosis of HCC and therefore, they might be considered among possible disease biomarkers ${ }^{[9]}$.

Epigenetic modifications are the changes occurring in the gene expression but do not involve changes in the DNA sequence. These modifications include DNA methylation and histone modifications. Interestingly, some enzymes involved in the epigenetic regulation have shown to be involved in HCC pathogenesis. These enzymes include RASSF1A, P16, DLC1 RhoA GTPase activating protein, runt related transcription factor 3 , and suppressor of cytocine signaling $1^{[11,12]}$. Multiple studies showed that (1) targeting these epigenetic modifiers might be effective in different types of cancers including HCC; and (2) they have the potential to be used as biomarkers for therapeutic response ${ }^{[13,14]}$. Histone deacetylase inhibitors such as panalinostat and belinostat have shown therapeutic efficacy in $\mathrm{HCC}^{[15,16]}$.

\section{CURRENTLY USED BIOMARKERS AND LIMITATIONS OF OLD BIOMARKERS}

\section{AFP for HCC surveillance in high-risk groups, diagnosis, and prognosis. Is it the ideal biomarker}

AFP is the most extensively used old biomarker. AFP is a common biomarker for the diagnosis and surveillance for HCC and it has reached the phase 5 of biomarker development stages (prospective randomized studies aiming to define the clinical utility of biomarkers).

AFP is a large serum glycoprotein that is a part of the serum albumin gene family ${ }^{[17]}$. The synthesis of AFP in the liver occurs during the fetal life is repressed during the adulthood ${ }^{[18]}$. Therefore, AFP levels often diminish rapidly after birth and remain low throughout the adulthood. However, AFP can be expressed under certain pathological conditions such as chronic liver disease, HCC, germ cell tumors, and gastric cancer $^{[19]}$.

There have been several investigations concerning the diagnostic utility of AFP suggesting that elevated serum AFP levels (> $20 \mathrm{ng} / \mathrm{mL}$ ) correlate with an increased risk for HCC development. Although the sensitivity of AFP is excellent, its specificity is low. The use of a higher cutoff value such as of $200 \mathrm{ng} / \mathrm{mL}$ drops the sensitivity 
to $22 \%$ while increases the specificity ${ }^{[20,21]}$. Therefore, the use of AFP in clinical practice is limited by the low sensitivity at cutoff values maintaining sufficiently high specificity.

Furthermore, it has not escaped our notice that the heterogeneity of AFP cut off values in the literature could be attributed to several epidemiological factors as the high incidence of HBV infection in Asian HCC patients and the fatty liver in Western countries. In addition, the control group in most of these studies includes subjects without HCC instead of subjects with suspected HCC to ensure prevalence rates comparable to the rates in clinical settings. Finally, several studies suffer from the "verification bias" because the reference standard (CT scan or MRI) had not been performed in all subjects to exclude tumor presence in non-HCC cases.

Although AFP can be used to help define the population at risk of $\mathrm{HCC}^{[22]}$, it has a suboptimal performance as a serological test for surveillance. The following study supports the use of AFP as a single biomarker for surveillance of HCC in particular populations or healthcare environments where ultrasound (US) is not available ${ }^{[23]}$. Furthermore, the use of US and AFP levels vs. the use of US alone offered additional detection in $6 \%-8 \%$ of HCC cases. The low specificity of AFP as a biomarker for HCC surveillance could be explained by (1) the transient rise in AFP levels in patients with cirrhosis reflecting an exacerbation of the hepatitis infection or in patients with chronic liver disease; and (2) the flares of underlying liver disease such as HBV, HCV or HCC development ${ }^{[24]}$.

Furthermore, AFP shows low sensitivity because it is not overexpressed in all HCC patients. It was found that elevated AFP levels were not evident in around $80 \%$ of small $\mathrm{HCCs}^{[25]}$. Only about $10 \%-20 \%$ of HCC tumors at the early stage can present with abnormal AFP serum levels. This observation has been recently found in a molecular subclass of aggressive HCCs (S2 class, EpCAM positive) ${ }^{[26-28]}$. In addition, AFP levels may be normal in up to $40 \%$ of patients diagnosed with early HCC. Based on data from the literature, a summary of the sensitivity and specificity of AFP is shown in Table $1^{[21]}$.

Another limitation of AFP includes the suboptimal performance in distinguishing intrahepatic cholangiocarcinoma and HCC. Although AFP (-) was the most sensitive assay for differentiating intrahepatic cholangiocarcinoma (ICC) from HCC (91.1\%), its specificity was significantly lower than other markers such as CA242 $(+)$ and carbohydrate antigen $(\mathrm{CA})$ CA19-9 $(+)^{[2]]}$.

This has a critical impact on the outcome of the misdiagnosed patients since surgical resection is generally the preferred therapeutic choice for HCC but not ICC $^{[30]}$. As a result, AFP has been excluded in some guidelines on HCC surveillance and diagnosis. In fact, the European Association for the Study of the Liver and the European Organization for Research and Treatment of Cancer (EASL-EORTC) clinical practice guidelines (CPG) ${ }^{[31]}$ for HCC screening and diagnosis do not include quantitative measurements of serum AFP and recommend surveillance by experienced personnel in the at-risk populations using abdominal US every 6 months. However, the American Association for the Study of Liver Diseases (AASLD) guidelines ${ }^{[32]}$ recommend surveillance by the US for cirrhotic adults every 6 months with optional use of AFP due to the poor sensitivity and specificity of this biomarker. Shorter follow-up interval (every 3-4 months) is recommended in case of any of these conditions: (1) a nodule of less than one $\mathrm{cm}$ has been detected; (2) after liver resection; or (3) after loco-regional therapy. In contrary, the follow-up 3-6 months of serum AFP was included in the diagnostic algorithm of hepatic nodules by the Oriental guidelines for HCC management. Therefore, in the most common guidelines, it is well established and recommended that US should a be part of surveillance and most commonly combined with AFP.

Furthermore, a recent systematic review on HCC surveillance ${ }^{[33]}$ showed that the most commonly used surveillance tests for HCC in cirrhotic patients were US and AFP. Four studies used only US for HCC surveillance, whereas the rest of studies used the combination of US and AFP at 6-month intervals. The 
Table 1. Cut off values for the commonly used biomarkers in the diagnosis of hepatocellular carcinoma; specificity and sensitivity characteristics

\begin{tabular}{lcc}
\hline Tumor marker & Specificity (\%) & Sensitivity (\%) \\
\hline AFP & {$[80-94]$} & {$[41-65]$} \\
$>20 \mathrm{ng} / \mathrm{mL}^{\star}$ & & \\
Elevated serum AFP-L3 & {$[83-94]$} & {$[37-75]$} \\
Range: $10 \%-35 \%$ & & \\
DCP/PVKA II & {$[70-100]$} & {$[41-89]$} \\
Range: $60-150 \mathrm{mAU} / \mathrm{mL}$ & & \\
\hline
\end{tabular}

*Higher cut off values increase specificity up to $100 \%$ and decrease sensitivity to less than $20 \%$. AFP: alpha-fetoprotein; AFP-L3: lens culinaris agglutinin-reactive fraction of alpha-fetoprotein; DCP: des- $\gamma$-carboxyprothrombin; PIVKA II: prothrombin induced by vitamin K absence

combined surveillance tests had better detection of early-stage HCC compared with no surveillance (OR 2.16, 95\% CI 1.80-2.6). On the other hand, the use of US only compared with no surveillance showed better detection of early stage HCC (OR 2.04, 95\% CI 1.55-2.68). Also, the use of either the US alone or in combination with AFP showed similar curative rates for the treatment (OR 2.23, 95\% CI 1.83-2.71 and 2.19, 95\% CI 1.89-2.53, respectively). Unfortunately, no studies have compared US alone $v s$. US in combination with AFP to detect early-stage HCC or to assess curative therapy. Regarding the improving survival, US plus AFP had a pooled risk ratio of 1.86 (95\% CI 1.76-1.97) whereas the US alone had a slightly lower pooled risk ratio of 1.75 (95\% CI 1.56-1.98). At present, it is unknown whether the addition of AFP allows for improved survival and which type of surveillance tests, US alone or in combination with AFP has a better-improved survival.

Furthermore, AFP has been incorporated in nomograms or calculators to predict the outcome of hepatic resection ${ }^{[34]}$ and transplantation ${ }^{[35]}$. AFP combined with SCCA might predict the risk of HCC in patients with chronic liver disease ${ }^{[36,37]}$. AFP level has been incorporated in many staging systems for HCC patients, such as Cancer of the Liver Italian Program, Chines University Prognostic Index, Groupe d'Etude et de Traitement du Carcinome Hépatocellulaire, and model to estimate survival in ambulatory HCC patients score ${ }^{[38]}$. Changes in AFP levels can predict the outcome in patients treated with transarterial chemoembolization ${ }^{[39]}$ or Sorafenib ${ }^{[40,41]}$.

Several limitations have been recognized in using AFP levels as a biomarker for HCC. First of all, AFP levels as a prognostic marker cannot help in therapeutic decisions and especially for patients with normal pretreatment AFP levels. Also, there is no consensus on when post-treatment AFP levels should be measured. Finally, the clinical utility of AFP response in patients treated with sorafenib has not yet been validated in prospective studies.

The limitations of AFP use highlight the need to identify novel biomarkers. Given the increasing incidence of HCC, it is necessary to explore whether other new or old serum biomarkers or a combination of them can compete with or complement that of the US and constitute an optimal performance in the diagnosis, prognosis, treatment response, and surveillance of $\mathrm{HCC}^{[9,42]}$.

\section{DCP}

Other serum markers such as DCP have also been explored, alone or in combination, in the diagnosis and surveillance of HCC.

DCP is an abnormal prothrombin molecule induced by vitamin $\mathrm{K}$ absence (PIVKA II) and posttranslational carboxylation machinery is known as DCP. DCP is an abnormal prothrombin molecule overproduced in HCC patients $^{[43,44]}$.

Unfortunately, DCP did not offer substantial advantages concerning AFP ${ }^{[45]}$. This marker is not used for early detection of HCC. DCP levels have been associated with portal vein invasion and advanced stage of HCC as 
with AFP-L3 fraction levels ${ }^{[46]}$. Serum DCP-based diagnosis showed suboptimal sensitivity (48\%-62\%) but satisfactory specificity $(81 \%-98 \%)$ in HCC patients ${ }^{[43,47]}$.

Regarding the role of PIVKA-II as treatment response marker, a recent meta-analysis showed that increased PIVKA-II levels could predict worsening overall survival and recurrence-free survival in patients with HCC who had curative ablation ${ }^{[48]}$. More studies are needed to confirm the clinical utility of PIVKA-II for HCC prognosis. At present, none of the above surveillance tests can be recommended to screen patients at high risk for HCC.

\section{Surveillance tests: the combination of AFP + AFP-L3 + DCP markers}

To address the problem of the markers being suboptimal due to sensitivity and specificity problems, combined application of DCP- and AFP-based biomarkers has been tested. The combination of AFP-L3, AFP and DCP markers used in 104 patients with HCC, 43\% of whom had AFP levels below $10 \mathrm{ng} / \mathrm{mL}$ achieved $60.6 \%$ sensitivity and $100 \%$ specificity ${ }^{[49]}$. In another large multicenter case-control study, DCP with AFP immunoassay for HCC detection showed increased sensitivity from $65 \%$ to $87 \%$, but specificity dropped from $84 \%$ to $69 \%{ }^{[50]}$. These studies supported the clinical utility of DCP for early-stage HCC diagnosis. However, further studies are needed to validate the effectiveness of DCP alone or as part of a new predictive score for HCC diagnosis. At present, the combination of AFP + AFP-L3 + DCP is included in the diagnostic algorithm of hepatic nodules by Oriental guidelines for HCC management, but not by Western guidelines.

\section{Other biomarkers}

In an effort to identify markers with highest sensitivity and specificity for the detection of HCC, many other molecules have been explored, including GPC3, GS, HSP70 (tissue), CK19, GP73, midkine, OPN, SCCA, Annexin A2, FGF3/4, miRNAs, lncRNA, CTCs, and cfDNA. Also, biomarkers obtained by proteomic-based approaches should be taken into account.

Proteomic studies have now identified multiple serum protein fragments with differential expression in HCC such as 70-kDa HSP70 and fructose-1, 6-bisphosphatase 1, the most consistently reported proteins, with upregulation and downregulation, respectively, in $\mathrm{HCC}^{[51]}$. Therefore, many of these proteins could serve as new biomarkers for HCC diagnosis, surveillance, prognosis, and treatment response. However, there is a limitation of proteomics currently used, the lack of agreement among various studies in reporting changes in protein expression associated with HCC.

Likewise, metabolomic studies investigate changes in lipid- and water-soluble metabolites found in the blood or urine. The scope was to identify a broader array of potential biomarkers for HCC ${ }^{[52-54]}$. Also, glycomic studies investigated $\mathrm{N}$-glycosylation patterns that may be associated with cancer development ${ }^{[55]}$. N-glycans are complex polysaccharides bound to biomolecules through $\mathrm{N}$-glycosylation and actively involved in several biological processes.

Also, genetic signatures can also be included among oncomarkers with prognostic meaning. Genomic variation between individuals has revealed multiple single nucleotide polymorphisms (SNPs) associated with HCC risk ${ }^{[56]}$. However, the high degree of change in gene expression based on patient ethnicity and underlying chronic liver disease makes it difficult to discover gene expression profiles that can reliably predict the risk of HCC.

In conclusion, many molecules have been explored as possible biomarkers for HCC, and some of them are described below.

\section{GPC3, GS, HSP70}

GPC3 is a cell-surface proteoglycan of the glypican family. This proteoglycan is overproduced in HCC cells and plays a pivotal role in regulating tumor growth. So, the soluble NH2-terminal fragment of GPC3 is 
used as a serological biomarker due to its ability to accurately distinguish between patients with small, welldifferentiated HCC tumors and those with cirrhosis ${ }^{[57]}$. This marker is similar to AFP, showed high specificity but low sensitivity ${ }^{[58]}$. Even if it is combined with miRNAs, such as miR-21, only a slight improvement in performance was shown compared with $\mathrm{AFP}^{[59]}$.

Other markers used in combination with GPC3 include HSP70 and GS. HSP70 belongs to a class of genes (heat shock proteins) abundantly overexpressed in advanced HCC as compared to early HCC, and in early HCC as compared to precancerous lesions ${ }^{[60]}$. Also, a study by Osada et al..$^{[61]}$ showed a stepwise increase in GS immunoreactivity from precancerous lesions to early and advanced HCC suggested that GS has a role in HCC. In CPG jointly published by EASL-EORTC recommend that GPC3 could be used alone or in combination with HSP70 and GS to distinguish well-differentiated HCC (early and grade 1) from dysplastic nodules of cirrhosis.

\section{NOVEL BIOMARKERS FOR HCC}

There is an unmet clinical need to discover better biomarkers for HCC that (1) fully correlate with the tumor stage; (2) can be detected in early HCC; and (3) allow for tumor surveillance and evaluation of therapeutic efficacy. Therefore, the research into novel HCC biomarkers continues. In this section, we briefly discuss the novel biomarkers for HCC, all are under investigation in clinical trials and are not currently used in clinical practice.

\section{CK19}

CK19 is a novel HCC biomarker associated with poor prognostic factors in HCC patients due to high risk of microvascular invasion and distant metastasis, as well as worse treatment outcome $e^{[62-64]}$.

\section{GP73}

GP73 is a transmembrane protein localized in the Golgi complex. Although it is absent in normal hepatocytes, abundantly overexpressed in HCC patients, compared with cirrhotic patients ${ }^{[65]}$. GP73 could be used as a marker in early-stage ${ }^{[66,67]}$.

\section{OPN}

OPN is a glycoprotein, an extracellular matrix protein ${ }^{[68]}$ expressed in HCC cells and other various types of malignancies ${ }^{[69]}$. OPN, although, it has a higher sensitivity in the discrimination of early HCC than AFP according to the clinical study of Shang et al ${ }^{[70]}$. The low specificity can be explained by its relationship with more than 30 types of cancers ${ }^{[7]]}$. Therefore a combination with AFP is necessary to optimize its performance ${ }^{[70]}$.

\section{SCCA}

SCCA is a serine protease inhibitor. It is found in squamous epithelium. The use of SCCA as an additional diagnostic marker with AFP for HCC has been well documented ${ }^{[72]}$. Also, it might play a role as a biomarker for response to treatment as there is an inverse correlation with the treatment response for HCC $^{[73]}$. Finally, the combination of AFP and SCCA should be investigated in future studies to validate the diagnostic role of SCCA as a predictor for the risk of HCC in patients with chronic liver disease.

\section{Annexin A2}

Annexin A2 is a calcium-dependent, phospholipid-binding protein. It is present in the cell surface, and it seems to be implicated in the development and metastasis of $\mathrm{HCC}^{[74]}$. It has been used as a serological biomarker for diagnosis and prognosis of early-stage HCC patients with higher sensitivity and specificity than $\mathrm{AFP}^{[75]}$.

\section{miRNA}

miRNAs are small non-coding endogenous RNAs that have been implicated in various biological roles at the cellular level including apoptosis and oncogenesis ${ }^{[76]}$. Some types of miRNAs act as controllers of different 
genes during HCC pathogenesis ${ }^{[77]}$. There are several types of miRNAs being tested as diagnostic and prognostic markers for HCC. To date, most common methods used for detection are the microarray, PCR and gene sequencing ${ }^{[78]}$. As a prognostic factor, low level and down-regulation of miRNA-542 and miRNA-139 are associated with poor prognosis as vascular invasion, larger tumor size and metastatic disease ${ }^{[7,80]}$. Expression of miRNA profile in the histopathological analysis after HCC resection can predict the risk of HCC recurrence within the Milan criteria ${ }^{[81]}$, HCC miRNAs expression varies between cirrhotic and non-cirrhotic $\mathrm{HCC}^{[82]}$. Remarkably, miRNAs like miR503HG suppress metastasis and inhibit malignant cell migration. Therefore, downregulation is associated with a higher risk of metastatic disease. This discovery may act as a template for future pharmacological targeted treatment ${ }^{[83]}$. Several studies compared them with the conventional HCC biomarkers such as AFP, DCP, AFP-L3 or used along with these biomarkers and the results demonstrated that a single miRNA or even better combination of different miRNAs were more sensitive than AFP, DCP, and AFP-L3\%. However, the miRNA expression profiles in HCC patients could vary significantly according to the tumor stages. Subsequently, it was difficult to distinguish between patients with different tumor stages. This was a limitation of the diagnostic utility of miRNAs as serological biomarkers.

Later, a panel of circulating miRNAs was developed, with the advances in miRNA screening techniques and the development of new bioinformatics tools achieved higher sensitivity and specificity in HCC diagnosis. Indeed, a miRNA panel, with cutoff $20 \mathrm{ng} / \mathrm{mL}$, showed better diagnostic sensitivity than AFP and similar specificity to AFP especially for the detection of small and early-stage tumors Also, this miRNA panel could be used as a prognostic score to improve the treatment outcome of HCC patients.

In conclusion, miRNAs are the promising biomarkers in the field of HCC diagnosis, prognosis, and potential therapeutic targets. However, they do not yet fit for the routine clinical setting.

\section{IncRNAs}

lncRNA are a unique class which are defined as transcripts of more than 200 nucleotides that present in genome-wide analysis of mammalian transcriptome. Accumulating evidence showed that dysregulated lncRNA had been involved in the pathogenesis of $\mathrm{HCC}^{[84,85]}$. Lately, IncRNA has been recognized as important regulators for carbohydrate and lipid metabolism; this has led to discovering a novel biomarker "IncRNA Ftx" which stimulate HCC progression and glycolysis. Therefore, IncRNA Ftx may act as a prototype for further research in targeted therapy for $\mathrm{HCC}^{[86]}$. A recent prospective study suggested combining lncRNA and AFP measurement may be a novel useful marker for HCC regarding diagnosis and $\operatorname{prognosis}^{[87]}$. Expression of RP11-466I1 in the serum and HCC tissue is associated with poor features like tumor capsule invasion ${ }^{[8]}$.

\section{CTCs}

One of the most adverse prognostic features of HCC is the presence of vascular invasion which leads to hematological spread and distant metastasis of malignant cells. Therefore, detection of CTCs has strategic clinical value in predicting HCC recurrence and monitoring treatment response ${ }^{[89,90]}$. Detection of CTCs is associated with poor overall survival and relapse-free survival ${ }^{[91]}$. In addition to that, CTCs positivity is significantly correlated with serum AFP level, vascular invasion and TNM stage which can reflect the histopathological status of $\mathrm{HCC}^{[92]}$. According to a recent meta-analysis of more than 20 studies, the CTC is not used as a sole indicator for diagnosis instead associated with poor clinical and pathological features ${ }^{[92]}$.

\section{cfDNA}

Dysregulated levels of cfDNA have a role in diagnosis, monitoring of treatment response, and even outcome prediction for cancer diseases ${ }^{[93-95]}$. Furthermore, single-nucleotide polymorphism of cfDNA such as Ser249 p53 mutation which is commonly found in the plasma DNA, unfortunately, is detected in HCC and nonHHC individuals ${ }^{[96]}$. However, differential methylation signatures identified in cfDNA, precede the occurrence 
of HCC, which are recommended to be used in combination with the conventional HCC biomarker AFP to improve the accuracy of HCC diagnosis because of lack of robustness ${ }^{[97,98]}$. Finally, the monitoring of cfDNA in the urine has been recently reported to be a promising tool to predict HCC recurrence ${ }^{[99]}$.

\section{Proteomic analysis and serum metabolite biomarkers}

An array of proteomic studies coupled with bioinformatics analysis identified serum protein fragments with differential expression in HCC, which possibly could serve as potential HCC biomarkers ${ }^{[100]}$. Luo et al ${ }^{[100]}$ investigated the utility of a serum metabolite biomarker panel of phenylalanyl-tryptophan and glycocholate. They found a higher diagnostic performance for the serum metabolite biomarkers compared with the AFP in terms of differentiating HCC from a high-risk population of cirrhosis.

\section{Core-fucosylated}

Core-fucosylated (CF) proteins could be candidate biomarkers in the diagnosis of $\mathrm{HCC}^{[101]}$. CF such as from fibronectin at site 1007 could differentiate HCC from cirrhosis in patients with alcoholic liver diseases. Also, CF cadherin-5 at site 61 could distinguish between HCC on chronic HCV hepatitis liver disease from cirrhosis. Furthermore, four differentially expressed apolipoprotein isoform proteins could differentiate NAFLD without cirrhosis form NAFLD-related cirrhosis or HCC on cirrhotic NAFLD ${ }^{[102]}$. Also, another protein, $\mathrm{CD} 5$ antigen-like, a soluble scavenger cysteine-rich protein that modulates inflammatory responses, could distinguish between NAFLD-related cirrhosis from NAFLD without cirrhosis but could not have any diagnostic value for HCC.

Finally, an 11-peak algorithm based on analysis of serum proteins was proven to be more accurate than several conventional biomarkers for early-stage $\mathrm{HCC}^{[103]}$. Overall, more proteomics probably will identify more HCC biomarkers.

\section{DEVELOPMENT OF NEW HCC BIOMARKERS FOR DIAGNOSIS, PROGNOSIS AND TUMOR RESPONSE PREDICTION}

So far, none of the new biomarkers outperform the conventional ones in such a way that it has been widely adopted in clinical practice. However, new data are promising.

\section{Biomarkers for HCC risk assessment}

Cirrhotic patients undergo justified periodical screenings to detect the early development of HCC. The identification of host factors such as the various biological pathways involved in liver carcinogenesis may help define specific adapted screening policies. Today, numerous candidate-gene studies have reported associations between SNPs and the presence of $\mathrm{HCC}^{[104]}$.

Unfortunately, the several host SNPs identified so far only partly explain the association with HCC in HCV-infected patients and did not enable good prediction on the individual and population levels ${ }^{[105]}$. It seems reasonable that various panels of SNPs should be incorporated into complex models of "genomic risk prediction", which take into account both host and environmental factors that can influence liver carcinogenesis at the near future.

At present, two biomarkers for HCC risk assessment have been developed including SNPs in germ-line epidermal growth factor associated with HCC on HCV-related cirrhosis and a specific 186-gene signature defining the high risk of HCC development in cirrhotic patients ${ }^{[106]}$.

\section{Biomarkers for HCC diagnosis}

Non-invasive diagnosis using EASL/AASLD criteria allows a confidential diagnosis of most HCCs above $2 \mathrm{~cm}^{[107]}$. However, imaging is less reliable in one-third of small nodules, and a liver biopsy is often indicated. In this 
scenario, EASL guidelines recommend testing the combination of 3 immunohistochemical markers (GPC3, HSP70 and GS). Besides, a 3-gene signature including GPC3, lymphatic vessel endothelial hyaluronan receptor 1, and survivin has also been proposed as an accurate molecular tool (sensitivity of $95 \%$ and specificity of $94 \%$ ) to discriminate dysplastic nodules and HCCs smaller than $2 \mathrm{~cm}$ in the setting of HCV etiology ${ }^{[108]}$. A step forward in the diagnosis of HCC could be provided by the development of a "liquid biopsy", i.e., the identification in the peripheral circulation of CTCs or circulating tumor DNA that have detached from a primary tumor ${ }^{[109]}$. A recent paper reported preliminary data in 8 tumor types, including $\mathrm{HCC}^{[110]}$.

\section{Prognostic biomarkers for HCC}

Regarding prognostic signatures for HCC, the phenotypic and molecular diversity of HCC allows us to identify several new biomarkers.

Changes in AFP levels have been used for prognostic stratification at a cut-off of $>500 \mathrm{ng} / \mathrm{dL}$ as a predictor of drop-out in the list of transplantation ${ }^{[3]}$ and as a predictor of the outcome of patients in phase III trials testing systemic therapies such as transarterial therapies or Sorafenib.

Furthermore, an excellent prognostic ability has also been reported for some genetic signatures obtained from tumor specimens in HCC patients treated by liver resection. Indeed, a 5-gene score based on the expression of TAF9, RAN, RAMP3, KRT19 and HN1 genes, represents the most reliable predictor of survival identified so far in multiple cohorts ${ }^{[111]}$. Also, neoangiogenesis-related genes (a panel of microRNA associated with regulation of angiogenesis) seem to be hallmarks of fast-growing HCCs and worst survival ${ }^{[12]}$. Finally, a 186-gene score from adjacent to tumor tissue was shown to have independent prognostic significance to predict overall survival in HCC patients ${ }^{[113]}$.

\section{The use of biomarkers as predictors of response to therapeutic targets to HCC}

The possibility of using novel biomarkers to predict tumor behavior to targeted therapies is appealing. Such biomarkers are the FGF that are essential pathway components of oncogenesis. FGF3/FGF4 amplification was found to predict increased response to the sorafenib in patients with $\mathrm{HCC}^{[114]}$. Sorafenib is a targeted therapy, classified as a tyrosine kinase inhibitor, has been the standard of care for patients with advanced HCC for the last decade ${ }^{[115]}$. Other predictive markers for sorafenib efficacy include high levels of soluble stem-cell factor receptor c-Kit and low levels of hepatocyte growth factor which have shown a non-significant trend for sorafenib efficacy ${ }^{[116,17]}$. Furthermore, patients with HCV-related HCC showed a higher benefit from sorafenib (HR: 0.47) compared to non-HCV patients (HR: 0.81) ${ }^{[118]}$.

Other targeted therapies for HCC include lenvaitinb ${ }^{[119]}$ and regorafenib ${ }^{[118]}$ as first-line treatments and cabozantinib $^{[120]}$ and ramucirumab ${ }^{[121]}$ as second-line treatments. In phase III REACH-2 trial, Zhu et al. . $^{[121]}$ demonstrated that ramucirumab as a second-line treatment achieved a significant and meaningful overall survival benefit with a favorable safety profile in HCC patients with baseline AFP greater than or equal to $400 \mathrm{ng} / \mathrm{mL}$, a population associated with poor prognosis; ramucirumab decreased mortality by $29 \% v s$. placebo as a second-line treatment for patients with advanced HCC and that AFP is a predictor of the efficacy of ramucirumab. Although AFP could predict the efficacy of ramucirumab, there is still a need for more biomarkers that show survival benefits for other HCC treatments ${ }^{[121]}$.

Another targeted drug for HCC, nivolumab, in a recent phase I-II clinical trial of 260 patients with advanced HCC has shown up to $16 \%$ of objective responses, some of them of long duration, obtaining a median overall survival of 16 months ${ }^{[122]}$. Again, the biomarkers used, the programmed death-1 and its ligand immunostaining status did not predict response to nivolumab ${ }^{[123]}$.

Recently, a gene signature capturing the immune class of HCC ( 30\% of patients) is currently under investigation as a treatment response predictor ${ }^{[24]}$. 


\section{HCC biomarkers that might be used as therapeutic targets}

As explained previously, GPC3 is a membrane-associated heparan sulfate proteoglycan that can be used as biomarker for HCC. However, recent studies have shown that it plays a role in cancer pathogenesis and proliferation and therefore it can be used as a therapeutic target to stop the progress and proliferation of tumor cells ${ }^{[125,126]}$.

Another biomarker and possible therapeutic target is the kinesin family member C1 (KIFC1). Recent studies showed that KIFC1 is overexpressed in HCC tumor tissues compared with non-tumor tissues, therefore, it might be used as a predictor for $\mathrm{HCC}^{[127]}$. Moreover, in vitro KIFC1 knockdown could effectively decrease the viability of HCC tumor cells, and induce apoptosis and cell death. This highlights that KIFC1 might be used as a biomarker and a therapeutic target for $\mathrm{HCC}^{[127]}$.

\section{Serum marker panels for HCC}

Aspartate aminotransferase to platelet ratio index

Aspartate aminotransferase to platelet ratio index (APRI) has been used to assess the risk of fibrosis and cirrhosis among hepatitis $\mathrm{C}$ patients. APRI has been recently investigated to predict the risk of cirrhosisdependent and independent HCC in HBV patients ${ }^{[128]}$. A recent study showed that APRI can predict response to transarterial chemoembolization treatment before starting the therapy ${ }^{[129]}$.

Fibrosis-4 index

A Korean study evaluating the role of Fibrosis-4 (FIB-4) index in predicting HCC among HBsAg positive individuals; they found that FIB-4 has a better predictive of HCC incidence, compared to that of ultrasonographic liver cirrhosis (C-index: 0.775 vs. $0.701 ; P=0.040)^{[130]}$. On the other hand, some reports show that liver fibrosis index (FIB-4) is not reliable for the prediction of $\mathrm{HCC}^{[131]}$.

\section{Forns test}

A study of liver fibrosis indices (APRI, FIB-4 index, and Forns index) showed that Forns index performed before HCV antiviral therapy was a predictor to identify patients with low likelihood of developing HCC after achieving a sustained virologic response ${ }^{[132]}$. Morevoer, Forns index was found to predict the recurrence and death of patients with hepatitis B-related HCC after curative resection ${ }^{[133]}$.

\section{CONCLUSION}

No biomarker combination is reliable enough to diagnose a lesion as HCC without confirmatory histological or radiological features. None of the new tumor markers outperform the conventional ones in such a way that it has been widely adopted in clinical practice. The diagnostic accuracy, particularly for early-stage HCC, can be improved by combining two or more biomarkers to reach an acceptable (> $80 \%)$ sensitivity with a modest decrement in specificity ${ }^{[49]}$. For this purpose, the accuracy can also be improved by measuring the overtime variability of the marker. However, all these proposals are waiting for prospective and external validations, and there are no recommended recall policies based on biomarker combinations or variability for the surveillance of patients at risk of developing HCC.

In terms of response to HCC treatment, AFP levels can predict response to ramucirumab treatment; an elevated AFP is a poor prognostic factor for ramucirumab survival benefit. However, future research should develop useful biomarkers for monitoring treatment activity, detecting early resistance to treatment and identifying patients who would more likely benefit from treatment.

\section{DECLARATIONS}

\section{Authors' contributions}

Reviewed the literature and wrote the manuscript: Zacharakis G, Aleid A, Aldossari KK 


\section{Availability of data and materials}

Not applicable.

\section{Financial support and sponsorship}

None.

\section{Conflicts of interest}

All authors declared that there are no conflicts of interest.

\section{Ethical approval and consent to participate}

Not applicable.

\section{Consent for publication}

Not applicable.

\section{Copyright}

(c) The Author(s) 2018.

\section{REFERENCES}

1. Global Burden of Disease Liver Cancer Collaboration, Akinyemiju T, Abera S, Ahmed M, Alam N, et al. The burden of primary liver cancer and underlying etiologies from 1990 to 2015 at the global, regional, and national level: results from the global burden of disease study 2015. JAMA Oncol 2017;3:1683-91.

2. Soerjomataram I, Lortet-Tieulent J, Parkin DM, Ferlay J, Mathers C, et al. Global burden of cancer in 2008: a systematic analysis of disability-adjusted life-years in 12 world regions. Lancet 2012;380:1840-50.

3. Parkin DM. The global health burden of infection-associated cancers in the year 2002. Int J Cancer 2006;118:3030-44.

4. Makarova-Rusher OV, Altekruse SF, McNeel TS, Ulahannan S, Duffy AG, et al. Population attributable fractions of risk factors for hepatocellular carcinoma in the United States. Cancer 2016;122:1757-65.

5. Fattovich G, Stroffolini T, Zagni I, Donato F. Hepatocellular carcinoma in cirrhosis: incidence and risk factors. Gastroenterology 2004; 127:S35-50.

6. Kanwal F, Hoang T, Kramer JR, Asch SM, Goetz MB, et al. Increasing prevalence of HCC and cirrhosis in patients with chronic hepatitis C virus infection. Gastroenterology 2011;140:1182-8.

7. El-Serag HB. Hepatocellular Carcinoma. N Engl J Med 2011;365:1118-27.

8. Zhang BH, Yang BH, Tang ZY. Randomized controlled trial of screening for hepatocellular carcinoma. J Cancer Res Clin Oncol 2004;130:417-22.

9. Chaiteerakij R, Zhang X, Addissie BD, Mohamed EA, Harmsen WS, et al. Combinations of biomarkers and Milan criteria for predicting hepatocellular carcinoma recurrence after liver transplantation. Liver Transpl 2015;21:559-606.

10. Zhu AX, Finn RS, Edeline J, Cattan S, Ogasawara S, et al. Pembrolizumab in patients with advanced hepatocellular carcinoma previously treated with sorafenib (KEYNOTE-224): a non-randomised, open-label phase 2 trial. Lancet Oncol 2018;19:940-52.

11. Umeda S, Kanda M, Kodera Y. Emerging evidence of molecular biomarkers in hepatocellular carcinoma. Histol Histopathol 2018;33:34355.

12. Khan FS, Ali I, Afridi UK, Ishtiaq M, Mehmood R. Epigenetic mechanisms regulating the development of hepatocellular carcinoma and their promise for therapeutics. Hepatol Int 2017;11:45-53.

13. Di Costanzo A, Del Gaudio N, Migliaccio A, Altucci L. Epigenetic drugs against cancer: an evolving landscape. Arch Toxicol 2014;88:165168.

14. Dhanak D, Jackson P. Development and classes of epigenetic drugs for cancer. Biochem Biophys Res Commun 2014;455:58-69.

15. Pathil A, Armeanu S, Venturelli S, Mascagni P, Weiss TS, et al. HDAC inhibitor treatment of hepatoma cells induces both TRAILindependent apoptosis and restoration of sensitivity to TRAIL. Hepatology 2006;43:425-34.

16. Yeo W, Chung HC, Chan SL, Wang LZ, Lim R, et al. Epigenetic therapy using belinostat for patients with unresectable hepatocellular carcinoma: a multicenter phase i/ii study with biomarker and pharmacokinetic analysis of tumors from patients in the Mayo Phase II Consortium and the Cancer Therapeutics Research Group. J Clin Oncol 2012;30:3361-7.

17. Terentiev AA, Moldogazieva NT. Alpha-fetoprotein: a renaissance. Tumor Biol 2013;34:2075-91.

18. Peterson ML, Ma C, Spear BT. Zhx2 and Zbtb20: novel regulators of postnatal alpha-fetoprotein repression and their potential role in gene reactivation during liver cancer. Semin Cancer Biol 2011;21:21-7.

19. Grizzi F, Colombo P, Taverna G, Chiriva-Internati M, Cobos E, et al. Geometry of human vascular system: is it an obstacle for quantifying antiangiogenic therapies? Appl Immunohistochem Mol Morphol 2007;15:134-9.

20. Gonzalez SA. Novel biomarkers for hepatocellular carcinoma surveillance: has the future arrived? Hepatobiliary Surg Nutr 2014;3:410-4.

21. Schütte K, Schulz C, Link A, Malfertheiner P. Current biomarkers for hepatocellular carcinoma: surveillance, diagnosis and prediction of prognosis. World J Hepatol 2015;7:139-49. 
22. Tsukuma H, Hiyama T, Tanaka S, Nakao M, Yabuuchi T, et al. Risk factors for hepatocellular carcinoma among patients with chronic liver disease. N Engl J Med 1993;328:1797-1801.

23. McMahon BJ, Bulkow L, Harpster A, Snowball M, Lanier A, et al. Screening for hepatocellular carcinoma in Alaska natives infected with chronic hepatitis B: a 16-year population-based study. Hepatology 2000;32:842-6.

24. Di Bisceglie AM, Sterling RK, Chung RT, Everhart JE, Dienstag JL, et al. Serum alpha-fetoprotein levels in patients with advanced hepatitis C: results from the HALT-C trial. J Hepatol 2005;43:434-41.

25. Bruix J, Sherman M; American Association for the Study of Liver Diseases. Management of hepatocellular carcinoma: an update. Hepatology 2011;53:1020-2.

26. Yamashita T, Budhu A, Forgues M, Wang XW. Activation of hepatic stem cell marker EpCAM by Wnt-beta-catenin signaling in hepatocellular carcinoma. Cancer Res 2007;67:10831-9.

27. Villanueva A, Minguez B, Forner A, Reig M, Llovet JM. Hepatocellular carcinoma: novel molecular approaches for diagnosis, prognosis, and therapy. Annu Rev Med 2010;61:317-28.

28. Hoshida Y, Nijman SM, Kobayashi M, Chan JA, Brunet JP, et al. Integrative transcriptome analysis reveals common molecular subclasses of human hepatocellular carcinoma. Cancer Res 2009;69:7385-92.

29. Tao LY, Cai L, He XD, Liu W, Qu Q. Comparison of serum tumor markers for intrahepatic cholangiocarcinoma and hepatocellular carcinoma. Am Surg 2010;76:1210-3.

30. Rimola J, Forner A, Reig M, Vilana R, de Lope CR, et al. Cholangiocarcinoma in cirrhosis: absence of contrast washout in delayed phases by magnetic resonance imaging avoids misdiagnosis of hepatocellular carcinoma. Hepatology 2009;50:791-8.

31. European Association For The Study Of The Liver; European Organisation For Research And Treatment Of Cancer. EASL-EORTC clinical practice guidelines: management of hepatocellular carcinoma. J Hepatol 2012;56:908-43.

32. Heimbach JK, Kulik LM, Finn RS, Sirlin CB, Abecassis MM, et al. AASLD guidelines for the treatment of hepatocellular carcinoma. Hepatology 2018;67:358-80.

33. Singal AG, Pillai A, Tiro J. Early detection, curative treatment, and survival rates for hepatocellular carcinoma surveillance in patients with cirrhosis: a meta-analysis. PLoS Med 2014;11:e1001624.

34. Li C, Shen JY, Zhang XY, Peng W, Wen TF, et al. Predictors of futile liver resection for patients with Barcelona clinic liver cancer stage B/C hepatocellular carcinoma. J Gastrointest Surg 2018;22:496-502.

35. Mazzaferro V, Sposito C, Zhou J, Pinna AD, De Carlis L, et al. Metroticket 2.0 model for analysis of competing risks of death after liver transplantation for hepatocellular carcinoma. Gastroenterology 2018;154:128-39.

36. Sangiovanni A, Del Ninno E, Fasani P, De Fazio C, Ronchi G, et al. Increased survival of cirrhotic patients with a hepatocellular carcinoma detected during surveillance. Gastroenterology 2004;126:1005-14.

37. Pontisso P, Quarta S, Caberlotto C, Beneduce L, Marino M, et al. Progressive increase of SCCA-IgM immune complexes in cirrhotic patients is associated with development of hepatocellular carcinoma. Int J cancer 2006;119:735-40.

38. Kinoshita A, Onoda H, Fushiya N, Koike K, Nishino H, et al. Staging systems for hepatocellular carcinoma: current status and future perspectives. World J Hepatol 2015;7:406-24.

39. Liu L, Zhao Y, Jia J, Chen H, Bai W, et al. The prognostic value of alpha-fetoprotein response for advanced-stage hepatocellular carcinoma treated with sorafenib combined with transarterial chemoembolization. Sci Rep 2016; doi: 10.1038/srep19851

40. Personeni N, Bozzarelli S, Pressiani T, Rimassa L, Tronconi MC, et al. Usefulness of alpha-fetoprotein response in patients treated with sorafenib for advanced hepatocellular carcinoma. J Hepatol 2012;57:101-7.

41. Nakazawa T, Hidaka H, Takada J, Okuwaki Y, Tanaka Y, et al. Early increase in $\alpha$-fetoprotein for predicting unfavorable clinical outcomes in patients with advanced hepatocellular carcinoma treated with sorafenib. Eur J Gastroenterol Hepatol 2013;25:683-9.

42. Tsuchiya N, Sawada Y, Endo I, Saito K, Uemura Y, et al. Biomarkers for the early diagnosis of hepatocellular carcinoma. World J Gastroenterol 2015;21:10573-83

43. Marrero JA, Su GL, Wei W, Emick D, Conjeevaram HS, et al. Des-gamma carboxyprothrombin can differentiate hepatocellular carcinoma from nonmalignant chronic liver disease in American patients. Hepatology 2003;37:1114-21.

44. Kumada T, Toyoda H, Tada T, Kiriyama S, Tanikawa M, et al. High-sensitivity Lens culinaris agglutinin-reactive alpha-fetoprotein assay predicts early detection of hepatocellular carcinoma. J Gastroenterol 2014;49:555-63.

45. Koike Y, Shiratori Y, Sato S, Obi S, Teratani T, et al. Des-gamma-carboxy prothrombin as a useful predisposing factor for the development of portal venous invasion in patients with hepatocellular carcinoma: a prospective analysis of 227 patients. Cancer 2001;91:561-9.

46. Sterling RK, Jeffers L, Gordon F, Sherman M, Venook AP, et al. Clinical utility of AFP-L3\% measurement in North American patients with HCV-related cirrhosis. Am J Gastroenterol 2007;102:2196-205.

47. Grizzi F, Franceschini B, Hamrick C, Frezza EE, Cobos E, et al. Usefulness of cancer-testis antigens as biomarkers for the diagnosis and treatment of hepatocellular carcinoma. J Transl Med 2007;5:3.

48. Zhang D, Liu Z, Yin X, Qi X, Lu B, et al. Prognostic value of PIVKA-II in hepatocellular carcinoma patients receiving curative ablation: a systematic review and meta-analysis. Int J Biol Markers 2018;33:266-74.

49. Marrero JA, Feng Z, Wang Y, Nguyen MH, Befeler AS, Roberts LR, Reddy KR, Harnois D, Llovet JM, Normolle D, Dalhgren J, Chia D, Lok AS, Wagner PD, Srivastava S, Schwartz M. Alpha-fetoprotein, des-gamma carboxyprothrombin, and lectin-bound alpha-fetoprotein in early hepatocellular carcinoma. Gastroenterology 2009;137:110-8.

50. Lok AS, Sterling RK, Everhart JE, Wright EC, Hoefs JC, et al. Des-gamma-carboxy prothrombin and alpha-fetoprotein as biomarkers for the early detection of hepatocellular carcinoma. Gastroenterology 2010;138:493-502.

51. Liu Z, Ma Y, Yang J, Qin H. Upregulated and downregulated proteins in hepatocellular carcinoma: a systematic review of proteomic profiling studies. OMICS 2011;15:61-71.

52. Budhu A, Roessler S, Zhao X, Yu Z, Forgues M, et al. Integrated metabolite and gene expression profiles identify lipid biomarkers associated with progression of hepatocellular carcinoma and patient outcomes. Gastroenterology 2013;144:1066-75. 
53. Beyoğlu D, Imbeaud S, Maurhofer O, Bioulac-Sage P, Zucman-Rossi J, et al. Tissue metabolomics of hepatocellular carcinoma: tumor energy metabolism and the role of transcriptomic classification. Hepatology 2013;58:229-38.

54. Fitian AI, Nelson DR, Liu C, Xu Y, Ararat M, et al. Integrated metabolomic profiling of hepatocellular carcinoma in hepatitis C cirrhosis through GC/MS and UPLC/MS-MS. Liver Int 2014;34:1428-44.

55. Ruhaak LR, Miyamoto S, Lebrilla CB. Developments in the identification of glycan biomarkers for the detection of cancer. Mol Cell Proteomics 2013;12:846-55.

56. Chen K, Shi W, Xin Z, Wang H, Zhu X, et al. Replication of genome wide association studies on hepatocellular carcinoma susceptibility loci in a chinese population. PLoS One 2013; doi: 10.1371/journal.pone.0077315.

57. Hippo Y, Watanabe K, Watanabe A, Midorikawa Y, Yamamoto S, et al. Identification of soluble NH2-terminal fragment of glypican-3 as a serological marker for early-stage hepatocellular carcinoma. Cancer Res 2004;64:2418-23.

58. Xu C, Yan Z, Zhou L, Wang Y. A comparison of glypican-3 with alpha-fetoprotein as a serum marker for hepatocellular carcinoma: a metaanalysis. J Cancer Res Clin Oncol 2013;139:1417-24

59. Tomimaru Y, Eguchi H, Nagano H, Wada H, Kobayashi S, et al. Circulating microRNA-21 as a novel biomarker for hepatocellular carcinoma. J Hepatol 2012;56:167-75.

60. Chuma M, Sakamoto M, Yamazaki K, Ohta T, Ohki M, et al. Expression profiling in multistage hepatocarcinogenesis: identification of HSP70 as a molecular marker of early hepatocellular carcinoma. Hepatology 2003;37:198-207.

61. Osada T, Sakamoto M, Nagawa H, Yamamoto J, Matsuno Y, et al. Acquisition of glutamine synthetase expression in human hepatocarcinogenesis: relation to disease recurrence and possible regulation by ubiquitin-dependent proteolysis. Cancer 1999;85:819-31.

62. Feng J, Zhu R, Chang C, Yu L, Cao F, et al. CK19 and glypican 3 expression profiling in the prognostic indication for patients with HCC after surgical resection. PLoS One 2016; doi: 10.1371/journal.pone.0151501.

63. Sun DW, Zhang YY, Sun XD, Chen YG, Qiu W, et al. Prognostic value of cytokeratin 19 in hepatocellular carcinoma: a meta-analysis. Clin Chim Acta 2015;448:161-9.

64. Lee JI, Lee JW, Kim JM, Kim JK, Chung HJ, et al. Prognosis of hepatocellular carcinoma expressing cytokeratin 19: comparison with other liver cancers. World J Gastroenterol 2012;18:4751-7.

65. Marrero JA, Romano PR, Nikolaeva O, Steel L, Mehta A, et al. GP73, a resident Golgi glycoprotein, is a novel serum marker for hepatocellular carcinoma. J Hepatol 2005;43:1007-12.

66. Tian L, Wang Y, Xu D, Gui J, Jia X, et al. Serological AFP/Golgi protein 73 could be a new diagnostic parameter of hepatic diseases. Int J Cancer 2011;129:1923-31.

67. Xu WJ, Guo BL, Han YG, Shi L, Ma WS. Diagnostic value of alpha-fetoprotein-L3 and Golgi protein 73 in hepatocellular carcinomas with low AFP levels. Tumor Biol 2014;35:12069-74.

68. Qin L. Osteopontin is a promoter for hepatocellular carcinoma metastasis: a summary of 10 years of studies. Front Med 2014;8:24-32.

69. Ramchandani D, Weber GF. Interactions between osteopontin and vascular endothelial growth factor: implications for cancer. Biochim Biophys Acta 2015;1855:202-22.

70. Shang S, Plymoth A, Ge S, Feng Z, Rosen HR, et al. Identification of osteopontin as a novel marker for early hepatocellular carcinoma. Hepatology 2012;55:483-90.

71. Weber GF. The cancer biomarker osteopontin: combination with other markers. Cancer Genomics and Proteomics 2011;8:263-88.

72. Giannelli G, Marinosci F, Sgarra C, Lupo L, Dentico P, et al. Clinical role of tissue and serum levels of SCCA antigen in hepatocellular carcinoma. Int J Cancer 2005;116:579-83.

73. Pozzan C, Cardin R, Piciocchi M, Cazzagon N, Maddalo G, et al. Diagnostic and prognostic role of SCCA-IgM serum levels in hepatocellular carcinoma (HCC). J Gastroenterol Hepatol 2014;29:1637-44.

74. Lokman NA, Ween MP, Oehler MK, Ricciardelli C. The role of annexin A2 in tumorigenesis and cancer progression. Cancer Microenviron 2011;4:199-208.

75. Sun Y, Gao G, Cai J, Wang Y, Qu X, et al. Annexin A2 is a discriminative serological candidate in early hepatocellular carcinoma. Carcinogenesis 2013;34:595-604.

76. Fu LL, Wen X, Bao JK, Liu B. MicroRNA-modulated autophagic signaling networks in cancer. Int J Biochem Cell Biol 2012;44:733-6.

77. Morishita A, Masaki T. miRNA in hepatocellular carcinoma. Hepatol Res 2015;45:128-41.

78. Borel F, Konstantinova P, Jansen PL. Diagnostic and therapeutic potential of miRNA signatures in patients with hepatocellular carcinoma. J Hepatol 2012;56:1371-83

79. Chen X, Zhang Q, Ma W, Lan T, Hong Z, et al. The abnormal expression of microRNA-542-3p in hepatocellular carcinoma and its clinical significance. Dis Markers 2018; doi: 10.1155/2018/3973250.

80. Wong CC, Wong CM, Tung EK, Au SL, Lee JM, et al. The microRNA miR-139 suppresses metastasis and progression of hepatocellular carcinoma by down-regulating rho-kinase 2. Gastroenterology 2011;140:322-31.

81. Sato F, Hatano E, Kitamura K, Myomoto A, Fujiwara T, et al. MicroRNA profile predicts recurrence after resection in patients with hepatocellular carcinoma within the Milan criteria. PLoS One 2011; doi: 10.1371/journal.pone.0016435.

82. Mei Y, You Y, Xia J, Gong JP, Wang YB. Identifying differentially expressed microRNAs between cirrhotic and non-cirrhotic hepatocellular carcinoma and exploring their functions using bioinformatic analysis. Cell Physiol Biochem 2018;48:1443-56.

83. Wang H, Liang L, Dong Q, Huan L, He J, et al. Long noncoding RNA miR503HG, a prognostic indicator, inhibits tumor metastasis by regulating the HNRNPA2B1/NF-kB pathway in hepatocellular carcinoma. Theranostics 2018;8:2814-29.

84. Carninci P, Kasukawa T, Katayama S, Gough J, Frith MC, et al. The transcriptional landscape of the mammalian genome. Science 2005;309:1559-63

85. Sun J, Bie B, Zhang S, Yang J, Li Z. Long non-coding RNAs: critical players in hepatocellular carcinoma. Int J Mol Sci 2014;15:20434-48.

86. Zhu X, Wu YB, Zhou J, Kang DM. Upregulation of IncRNA MEG3 promotes hepatic insulin resistance via increasing FoxO1 expression. Biochem Biophys Res Commun 2016;469:319-25. 
87. Xu H, Dong X, Chen Y, Wang X. Serum exosomal hnRNPH1 mRNA as a novel marker for hepatocellular carcinoma. Clin Chem Lab Med 2018;56:479-84.

88. Zhang J, Zhang D, Zhao Q, Qi J, Li X, et al. A distinctively expressed long noncoding RNA, RP11-466I1.1, may serve as a prognostic biomarker in hepatocellular carcinoma. Cancer Med 2018; doi: 10.1002/cam4.1565.

89. Imamura $\mathrm{H}$, Matsuyama $\mathrm{Y}$, Tanaka E, Ohkubo $\mathrm{T}$, Hasegawa $\mathrm{K}$, et al. Risk factors contributing to early and late phase intrahepatic recurrence of hepatocellular carcinoma after hepatectomy. J Hepatol 2003;38:200-7.

90. Xu W, Cao L, Chen L, Li J, Zhang XF, et al. Isolation of circulating tumor cells in patients with hepatocellular carcinoma using a novel cell separation strategy. Clin Cancer Res 2011;17:3783-93.

91. Fan JL, Yang YF, Yuan CH, Chen H, Wang FB. Circulating tumor cells for predicting the prognostic of patients with hepatocellular carcinoma: a meta analysis. Cell Physiol Biochem 2015;37:629-40.

92. Sun C, Liao W, Deng Z, Li E, Feng Q, et al. The diagnostic value of assays for circulating tumor cells in hepatocellular carcinoma: a metaanalysis. Medicine (Baltimore) 2017; doi: 10.1097/MD.0000000000007513.

93. Leon SA, Shapiro B, Sklaroff DM, Yaros MJ. Free DNA in the serum of cancer patients and the effect of therapy. Cancer Res 1977;37:64650 .

94. Swystun LL, Mukherjee S, Liaw PC. Breast cancer chemotherapy induces the release of cell-free dna, a novel procoagulant stimulus. J Thromb Haemost 2011;9:2313-21.

95. García-Olmo DC, Domínguez C, García-Arranz M, Anker P, Stroun M, et al. Cell-free nucleic acids circulating in the plasma of colorectal cancer patients induce the oncogenic transformation of susceptible cultured cells. Cancer Res 2010;70:560-7.

96. Kirk GD, Lesi OA, Mendy M, Szymañska K, Whittle H, et al. 249(ser) TP53 mutation in plasma DNA, hepatitis B viral infection, and risk of hepatocellular carcinoma. Oncogene 2005;24:5858-67.

97. Liao W, Mao Y, Ge P, Yang H, Xu H, et al. Value of quantitative and qualitative analyses of circulating cell-free DNA as diagnostic tools for hepatocellular carcinoma: a meta-analysis. Medicine (Baltimore) 2015; doi: 10.1097/md.0000000000000722.

98. Shi M, Chen MS, Sekar K, Tan CK, Ooi LL, et al. A blood-based three-gene signature for the non-invasive detection of early human hepatocellular carcinoma. Eur J Cancer 2014;50:928-36.

99. Hann HW, Jain S, Park G, Steffen JD, Song W, et al. Detection of urine DNA markers for monitoring recurrent hepatocellular carcinoma. Hepatoma Res 2017;3:105-11.

100. Luo P, Yin P, Hua R, Tan Y, Li Z, et al. A large-scale, multicenter serum metabolite biomarker identification study for the early detection of hepatocellular carcinoma. Hepatology 2017; doi: 10.1002/hep.29561.

101. Yin H, Tan Z, Wu J, Zhu J, Shedden KA, et al. Mass-selected site-specific core-fucosylation of serum proteins in hepatocellular carcinoma. J Proteome Res 2015;14:4876-84.

102 Gray J, Chattopadhyay D, Beale GS, Patman GL, Miele L, et al. A proteomic strategy to identify novel serum biomarkers for liver cirrhosis and hepatocellular cancer in individuals with fatty liver disease. BMC Cancer 2009;9:271.

103. Zinkin NT, Grall F, Bhaskar K, Otu HH, Spentzos D, et al. Serum proteomics and biomarkers in hepatocellular carcinoma and chronic liver disease. Clin Cancer Res 2008;14:470-7.

104. Nahon P, Zucman-Rossi J. Single nucleotide polymorphisms and risk of hepatocellular carcinoma in cirrhosis. J Hepatol 2012;57:663-74.

105. Walker AJ, Peacock CJ, Pedergnana V; STOP-HCV Consortium, Irving WL. Host genetic factors associated with hepatocellular carcinoma in patients with hepatitis C virus infection: a systematic review. J Viral Hepat 2018;25:442-56.

106. Hoshida Y, Villanueva A, Sangiovanni A, Sole M, Hur C, et al. Prognostic gene-expression signature for patients with hepatitis C-related early-stage cirrhosis. Gastroenterology 2013;144:1024-30.

107. Llovet JM, Zucman-Rossi J, Pikarsky E, Sangro B, Schwartz M, et al. Hepatocellular carcinoma. Nat Rev Dis Primers 2016;2:16018.

108. Llovet JM, Chen Y, Wurmbach E, Roayaie S, Fiel MI, et al. A molecular signature to discriminate dysplastic nodules from early hepatocellular carcinoma in HCV cirrhosis. Gastroenterology 2006;131:1758-67.

109. Han X, Wang J, Sun Y. Circulating tumor DNA as biomarkers for cancer detection. Genomics Proteomics Bioinformatics 2017;15:59-72.

110. Cabel L, Proudhon C, Buecher B, Pierga JY, Bidard FC. Circulating tumor DNA detection in hepatocellular carcinoma. Ann Oncol 2018;29:1094-6.

111. Nault JC, De Reyniès A, Villanueva A, Calderaro J, Rebouissou S, et al. A hepatocellular carcinoma 5-gene score associated with survival of patients after liver resection. Gastroenterology 2013;145:176-87.

112. Villa E, Critelli R, Lei B, Marzocchi G, Cammà C, et al. Neoangiogenesis-related genes are hallmarks of fast-growing hepatocellular carcinomas and worst survival. Results from a prospective study. Gut 2016;65:861-9.

113. Hoshida Y, Villanueva A, Kobayashi M, Peix J, Chiang DY, et al. Gene expression in fixed tissues and outcome in hepatocellular carcinoma. N Engl J Med 2008;359:1995-2004.

114. Arao T, Ueshima K, Matsumoto K, Nagai T, Kimura H, et al. FGF3/FGF4 amplification and multiple lung metastases in responders to sorafenib in hepatocellular carcinoma. Hepatology 2013;57:1407-15.

115. Bruix J, Cheng AL, Meinhardt G, Nakajima K, De Sanctis Y, et al. Prognostic factors and predictors of sorafenib benefit in patients with hepatocellular carcinoma: analysis of two phase III studies. J Hepatol 2017;67:999-1008.

116. Llovet JM, Villanueva A, Lachenmayer A, Finn RS. Advances in targeted therapies for hepatocellular carcinoma in the genomic era. Nat Rev Clin Oncol 2015;12:408-24.

117. Llovet JM, Peña CE, Lathia CD, Shan M, Meinhardt G, et al. Plasma biomarkers as predictors of outcome in patients with advanced hepatocellular carcinoma. Clin Cancer Res 2012;18:2290-300.

118. Bruix J, Qin S, Merle P, Granito A, Huang YH, et al. Regorafenib for patients with hepatocellular carcinoma who progressed on sorafenib treatment (RESORCE): a randomised, double-blind, placebo-controlled, phase 3 trial. Lancet 2017;389:56-66.

119. Kudo M, Finn RS, Qin S, Han KH, Ikeda K, et al. Lenvatinib versus sorafenib in first-line treatment of patients with unresectable hepatocellular carcinoma: a randomised phase 3 non-inferiority trial. Lancet 2018;391:1163-73. 
120. Abou-Alfa GK, Meyer T, Cheng AL, El-Khoueiry AB, Rimassa L, et al. Cabozantinib in patients with advanced and progressing hepatocellular carcinoma. N Engl J Med 2018;379:54-63.

121. Zhu AX, Kang YK, Yen CJ, Finn RS, Galle PR, et al. REACH-2: a randomized, double-blind, placebo-controlled phase 3 study of ramucirumab versus placebo as second-line treatment in patients with advanced hepatocellular carcinoma (HCC) and elevated baseline alpha-fetoprotein (AFP) following first-line sorafe. J Clin Oncol 2018;36:4003.

122. El-Khoueiry AB, Sangro B, Yau T, Crocenzi TS, Kudo M, et al. Nivolumab in patients with advanced hepatocellular carcinoma (CheckMate 040): an open-label, non-comparative, phase 1/2 dose escalation and expansion trial. Lancet 2017;389:2492-502.

123. Maleki Vareki S, Garrigós C, Duran I. Biomarkers of response to PD-1/PD-L1 inhibition. Crit Rev Oncol Hematol 2017;116:116-24.

124. Sia D, Jiao Y, Martinez-Quetglas I, Kuchuk O, Villacorta-Martin C, et al. Identification of an immune-specific class of hepatocellular carcinoma, based on molecular features. Gastroenterology 2017;153:812-26.

125. Wang L, Yao M, Pan LH, Qian Q, Yao DF. Glypican-3 is a biomarker and a therapeutic target of hepatocellular carcinoma. Hepatobiliary Pancreat Dis Int 2015;14:361-6.

126. Filmus J, Capurro M. Glypican-3: a marker and a therapeutic target in hepatocellular carcinoma. FEBS J 2013;280:2471-6.

127. Fu X, Zhu Y, Zheng B, Zou Y, Wang C, et al. KIFC1, a novel potential prognostic factor and therapeutic target in hepatocellular carcinoma. Int J Oncol 2018;52;1912-22.

128. Hann HW, Wan S, Lai Y, Hann RS, Myers RE, et al. Aspartate aminotransferase to platelet ratio index as a prospective predictor of hepatocellular carcinoma risk in patients with chronic hepatitis B virus infection. J Gastroenterol Hepatol 2015;30:131-8.

129. Tang T, Qiu JL, Li GW, Huang MP, Li Y, et al. Aspartate aminotransferase-to-platelet ratio predicts response to transarterial chemoembolisation and prognosis in hepatocellular carcinoma patients. Clin Radiol 2018;73:259-65.

130. Suh B, Park S, Shin DW, Yun JM, Yang HK, et al. High liver fibrosis index FIB-4 is highly predictive of hepatocellular carcinoma in chronic hepatitis B carriers. Hepatology 2015;61:1261-8.

131. Demir M, Grünewald F, Lang S, Schramm C, Bowe A, et al. Elevated liver fibrosis index FIB-4 is not reliable for HCC risk stratification in predominantly non-Asian CHB patients. Medicine (Baltimore) 2016; doi: 10.1097/MD.0000000000004602.

132. Toyoda H, Tada T, Tachi Y, Hirai T, Yasuda S, et al. Liver fibrosis indices for identifying patients at low risk of developing hepatocellular carcinoma after eradication of HCV. Antivir Ther 2017;22:185-93.

133. Choi WM, Lee JH, Ahn H, Cho H, Cho YY, et al. Forns index predicts recurrence and death in patients with hepatitis B-related hepatocellular carcinoma after curative resection. Liver Int 2015;35:1992-2000. 